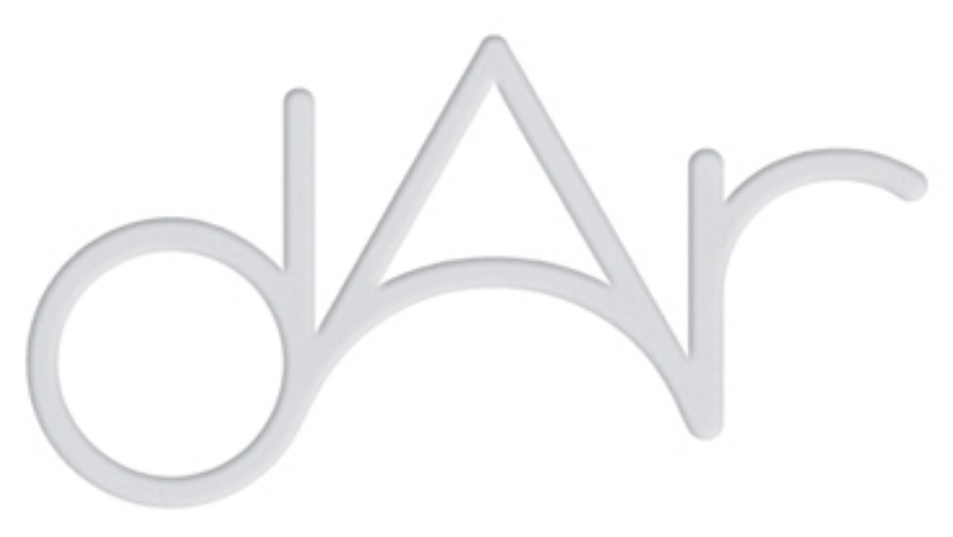

\title{
Le tourisme culturel-patrimonial: une contribution à la valorisation des villages kabyles du Djurdjura
}
Autor(es):
Frendi, Karima; Ait-Ihadj, Zoulikha
Publicado por: Imprensa da Universidade de Coimbra
URL
persistente:
URI:http://hdl.handle.net/10316.2/44432
DOI:
DOI:https://doi.org/10.14195/2182-844X_5_11
Accessed : $\quad$ 26-Apr-2023 08:06:54

A navegação consulta e descarregamento dos títulos inseridos nas Bibliotecas Digitais UC Digitalis, UC Pombalina e UC Impactum, pressupõem a aceitação plena e sem reservas dos Termos e Condições de Uso destas Bibliotecas Digitais, disponíveis em https://digitalis.uc.pt/pt-pt/termos.

Conforme exposto nos referidos Termos e Condições de Uso, o descarregamento de títulos de acesso restrito requer uma licença válida de autorização devendo o utilizador aceder ao(s) documento(s) a partir de um endereço de IP da instituição detentora da supramencionada licença.

Ao utilizador é apenas permitido o descarregamento para uso pessoal, pelo que o emprego do(s) título(s) descarregado(s) para outro fim, designadamente comercial, carece de autorização do respetivo autor ou editor da obra.

Na medida em que todas as obras da UC Digitalis se encontram protegidas pelo Código do Direito de Autor e Direitos Conexos e demais legislação aplicável, toda a cópia, parcial ou total, deste documento, nos casos em que é legalmente admitida, deverá conter ou fazer-se acompanhar por este aviso.

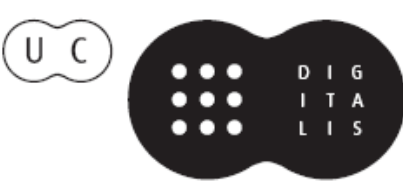



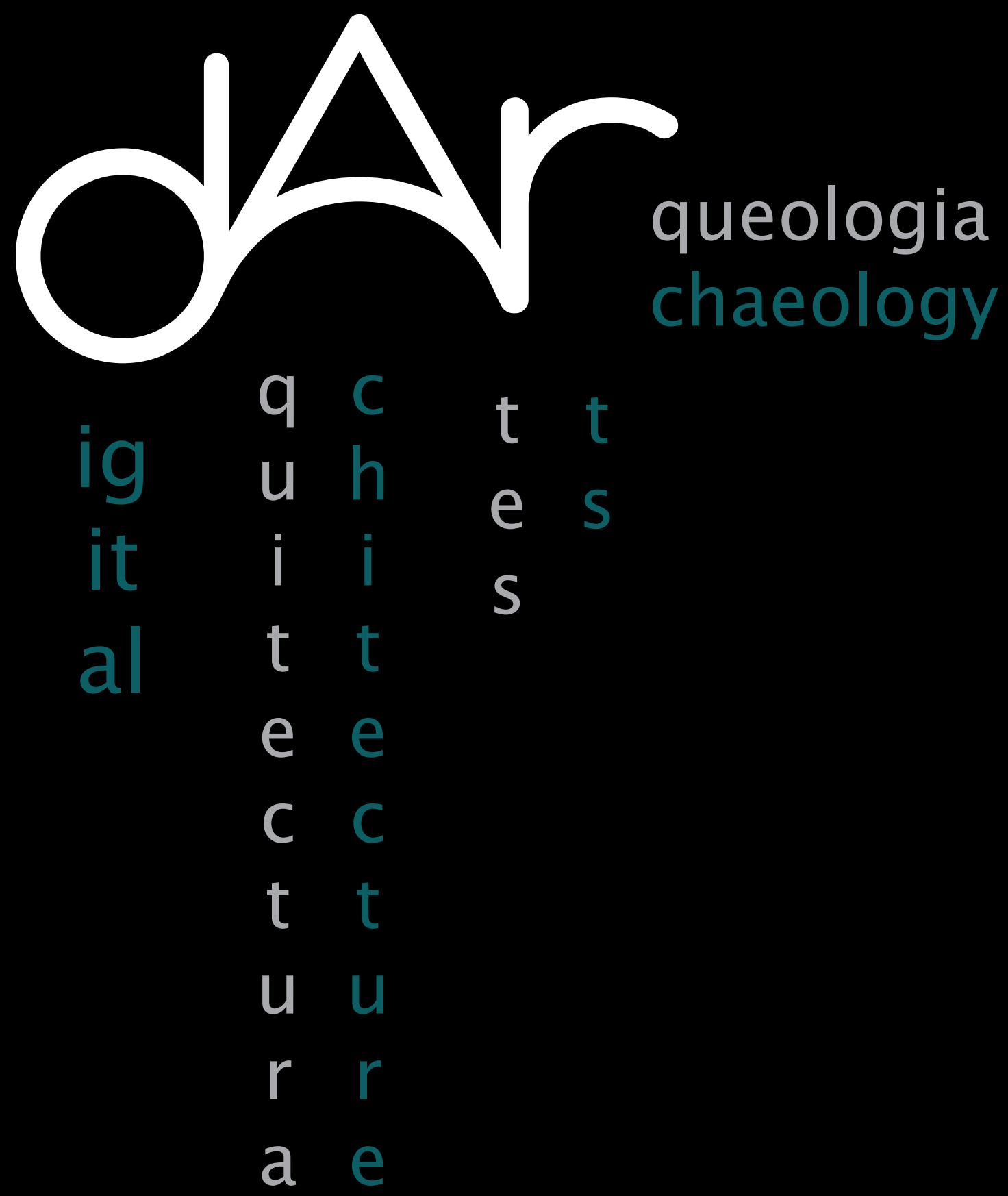

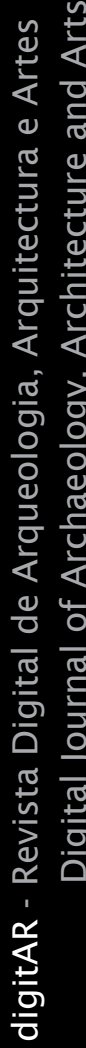




\title{
LE TOURISME CULTUREL-PATRIMONIAL, UNE CONTRIBUTION À LA VALORISATION DES VILLAGES KABYLES DU DJURDJURA
}

\author{
Karima Frendi ${ }^{1}$, Zoulikha Ait-lhadj ${ }^{2}$ \\ ${ }^{1}$ Architecte principale, Wilaya de Tizi-Ouzou/Algérie \\ ${ }^{2}$ Doctorante, Université Mouloud Mammeri de Tizi-Ouzou
}

\section{Résumé}

La préoccupation liée à la préservation, la sauvegarde et la valorisation du village kabyle traditionnel en Algérie, comme témoignage de notre histoire et symbole de notre identité, se chevauche inévitablement avec celle du développement local des régions de la zone de montagne, notamment du Djurdjura. Actuellement, ses villages se dépeuplent vue l'absence d'alternative de développement, ce qui les faits sombrer dans l'oubli et la désuétude, "cas du village traditionnel d'Ath El Kaid, situé au cœur de la montagne du Djurdjura, malgré qu'il soit érigé en secteurs sauvegardés après son classement comme patrimoine culturel national année 2009».

En vue d'une valorisation du village traditionnel, il est crucial d'opter pour sa redynamisation, et d'en faire un élément actif dans son territoire. Ainsi, l'intégration d'une activité touristique mobilisant ses potentialités naturelles et culturelles s'imposent, pour un développement local certain. Le tourisme culturel-patrimonial est une forme de tourisme qui a pour but de découvrir le patrimoine culturel et architectural d'une région. Il doit être réfléchi comme un agent de développement durable, et s'appliquer aux valeurs fondamentales à savoir l'authenticité des projets et la protection du patrimoine.

Le tourisme culturel-patrimonial, peut contribuer de façon significative à la prospérité économique de la région de la Kabylie du Djurdjura, comme il peut générer des retombées sociales à savoir la reconnaissance de son patrimoine culturel et architectural comme composante du territoire. Et par conséquent la promotion de sa culture locale vivante, la découverte des différentes activités et traditions de la population locale. Ainsi les

https://doi.org/10.14195/2182-844X_5_11 
apports de ce type de tourisme, contribuent à améliorer la qualité de vie des habitants et leur procurent un sentiment de fierté renouvelé à l'égard de leur culture et leur patrimoine et deviennent soucieux de leur préservation à travers le temps.

Mots clés: Zone de montagne, village Kabyle traditionnel, tourisme culturel-patrimonial, développement local, développement durale.

\section{Introduction}

Le patrimoine est défini comme étant un héritage qui préserve les liens intergénérationnels. Il est le facteur d'identité pour un pays, une région et une communauté. Il est au départ, celui des grands monuments, une notion d'abord étroite qui s'est progressivement élargie ; pour s'étendre à l'ensemble des biens culturels, des traditions, des modes de vies, et de tout ce qui peut constituer la culture de l'homme. L'apparition d'un nouveau paradigme de développement économique dans lequel le territoire constitue la principale composante, implique un nouveau regard sur la notion de patrimoine lui conférant un rôle moteur dans le développement économique, culturel, et social.

La prise de conscience croissante à l'égard de la conservation de notre patrimoine culturel en Algérie a induit une patrimonialisation d'un legs ancestral important de la société traditionnelle. "Le village kabyle » est l'un de ces importants héritages incarnant un témoignage authentique essentiel pour notre mémoire collective, qui suscite une reconstitution et une transmission authentique aux générations à venir, comme témoignage de notre culture porteuse de signification et de diversité, un atout indispensable pour réduire la pauvreté et parvenir au développement territorial durable. Ceci nous interpelle à nous interroger sur les défis et les perspectives de la gestion du patrimoine local dans le développement du tourisme. Par conséquent : Le tourisme culturelpatrimonial est-il la panacée salvatrice capable de redynamiser la vie socio-économique locale, et motrice de sauvegarde de l'identification patrimoniale? Et quels sont les formes de mise en tourisme des éléments du patrimoine essentiellement de zone de montagne capables d'enclencher une dynamique de développement socio-économique?

L'objectif de notre propos vise à exalter le potentiel patrimonial de la zone de montagne kabyle, notamment l'héritage de la société 
traditionnelle par ses villages traditionnels y compris toutes les valeurs intangibles qui y sont incarnées, et ce par le biais d'un recensement des ressources patrimoniales potentielles susceptibles d'être mobilisées dans un processus de valorisation touristique pour la région particulièrement et par conséquent pour l'Algérie toute entière qui, dans la conjoncture actuelle, suite à l'effondrement des rentes pétrolières, le pays doit impérativement améliorer et particulariser son offre touristique devenue première industrie mondiale.

Ce processus de valorisation touristique, à notre sens, pourrait garantir la sauvegarde et la pérennité de cet héritage et stimuler ainsi sa conservation. De ce fait, notre contribution interroge la relation entre le patrimoine et sa mise en tourisme et leurs effets sur le développement local.

\section{Le patrimoine matériel et immatériel facteur de développement ter- ritorial}

Le patrimoine essentiellement matériel a été considéré pour longtemps comme objet improductif et une source de dépense. De nos jours, il est plutôt perçu comme créateur de richesses et facteur d'attractivité pour les territoires. Le patrimoine matériel ne peut être une ressource territoriale valorisante que s'il est investi de valeurs symboliques, de ce fait, la reconnaissance et la préservation du patrimoine matériel sont tributaires de la préservation du patrimoine immatériel. Ce changement de regard non négligeable affirme le rôle crucial du patrimoine dans le développement économique, culturel et social. En réalité, le patrimoine est une ressource capable de générer des activités multiples mobilisant différents acteurs territoriaux, ces activités sont souvent étroitement liées au tourisme, un secteur reconnu comme créateur d'emplois et promoteur de produit locaux. Il constitue une issue pour sortir de la crise pour les territoires vulnérables économiquement.

\section{Richesse et importance du patrimoine en zone de montagne}

Les zones de montagne sont généralement inaccessibles, fragiles et souvent ignorées dans les prises de décisions. Elles abritent les populations les plus pauvres du monde. En Algérie, elles sont essentiellement situées dans la région Nord du pays et occupent $60 \%$ de la superficie pour $20 \%$ de la population totale. Ces zones sont d'une importance indéniable au vu de leurs potentialités (naturelles, socioculturelles, leur diversité 
climatique, géologique et biologique, la variété de leurs paysages, traditions culturelles et pratiques traditionnelles qui les caractérisent). Néanmoins, le manque d'infrastructures et d'équipements ainsi que le déficit en termes d'investissement ont engendré une migration de la population locale induisant ainsi une dégradation du patrimoine naturel et culturel.

La zone de montagne Kabyle est classée par la législation algérienne en vigueur comme territoire rural. Ces territoires ruraux disposent d'un patrimoine naturel, culturel et paysager composé tout à la fois d'éléments matériels et immatériels. Ce patrimoine est devenu aujourd'hui dans bon nombre de pays, en particulier au Maroc, un moteur et un facteur de développement à travers les politiques initiées pour sa valorisation. Sa préservation est donc jugée primordiale et donne un sens à l'évolution du monde rural et de la zone de montagne en particulier. La mise en valeur de ce patrimoine et sa protection s' avèrent donc indispensables au maintien des équilibres écologiques et au développement économique, social et culturel. [Ahmed Saber, 2008] avance que la loi 04-03 du 23 juin 2004 relative à la protection des zones de montagnes dans le cadre du développement durable met en place une nouvelle approche des zones de montagnes par "Massif montagneux » et elle est principalement fondée sur la structuration et l'organisation de l'espace montagnard, la durabilité des ressources naturelles, la promotion de la multi-activité et de l'emploi dans le but d'améliorer les conditions socio-économiques des communautés locales.

\section{Développement local et patrimoine}

Xavier Greffe, 1984 définit le développement local comme un processus de diversification et d'enrichissement des activités économiques et sociales sur un territoire, qui est le produit des efforts de sa population intégrant ses composantes économiques, sociales et culturelles (réf auteur, année). Le projet de développement fera d'un espace de contigüité, un espace de solidarité active dont les outils de base sont fondés sur l'aménagement du territoire qui fixe le cadre de développement des zones, la politique de décentralisation, la gouvernance locale orientée vers la définition d'un projet global commun et spécifique, la participation citoyenne et le financement diversifié.

En terme général, le développement local est une stratégie de développement orienté vers l'action qui valorise les potentialités et richesses locales, et mise sur les acteurs locaux en profitant de la 
dynamique qui les anime. L'objectif du développement local vise à intégrer ces acteurs locaux de façon participative dans l'élaboration de plans locaux de développement en accord avec les grandes orientations politico-économiques du pays dans le but d'améliorer la qualité de leur vie. Le développement local dans la zone de montagne, convient à l'exploitation et la valorisation durable de ses atouts. Par conséquent, le patrimoine " rural » qui a été longtemps traité avec légèreté et mépris, considéré comme 'parent pauvre' du patrimoine au profit des monuments de prestige fortement privilégiés, revêt un intérêt particulier selon Michael Dower : "il représente à la fois la mémoire collective de la population et une ressource essentielle pour son avenir. Sa protection et sa mise en valeur ne va pas à l'encontre de la satisfaction des besoins présents, bien au contraire l'importance du patrimoine, même lorsqu'il est modeste, fait l'objet d'un consensus de plus en plus large. Le défi aujourd'hui est plutôt de mieux intégrer la protection, la mise en valeur et la valorisation du patrimoine dans l'approche locale du développement. Défenseurs du patrimoine et acteurs du développement local sont des partenaires ». Le développement du tourisme constitue un moyen concret pour une mise en valeur durable des zones de montagnes et de leur potentiel patrimonial sachant que leurs ressources économiques nécessaires au développement sont limitées.

\section{Tourisme et identités locales au prisme de la durabilité}

Le tourisme, de nos jours, constitue une expérience importante dans le processus efficace du développement local. Plusieurs recherches et contributions d'auteurs ont concerné le tourisme fondé sur un développement culturellement réussi, écologiquement adéquat et économiquement rentable, qui contribue à une société durable. Ces auteurs ont essayé de réfléchir aux conditions dans lesquelles le tourisme peut être un facteur de développement local tout en protégeant les écosystèmes. D'autres auteurs pensent que le tourisme nuit au patrimoine, qui se manifeste dans le pillage des vestiges, et entraîne d'inévitables mutations du patrimoine qui «tend à passer d'un patrimoine identitaire à un paysage décor ou folklore à valeur touristique marchande " [Boujrouf, 2004, p.65]. Il est vrai que l'activité touristique n'est pas sans risque pour le patrimoine, il est extrêmement important d'agir en faveur d'un tourisme " plus respectueux des cultures locales». La protection du patrimoine doit permettre aux membres des communautés d'accueil et aux visiteurs de vivre et de comprendre le patrimoine et la culture de cette communauté de façon concrète, grâce à une offre touristique 
responsable et bien administrée. Le tourisme en zone de montagnes illustre un dilemme entre conservation et développement. Les politiques touristiques applicables au patrimoine culturel doivent alors dériver des politiques de conservation afin de préserver les valeurs patrimoniales de ses paysages.

Selon un des principes de la charte du tourisme culturel de l'ICOMOS (1999) : " la relation entre le tourisme et le patrimoine est dynamique et doit être gérée d'une manière durable au profit des générations actuelles et futures ", ceci suscite une imposition de limites au type ainsi qu'à l'intensité des activités touristiques en zone de montagnes et en lieux de patrimoine, et afin que les avantages touchent un grand nombre de personnes, il est impératif de mettre en place un mécanisme basé sur la surveillance, la réglementation, et le contrôle des activités qui pourraient compromettre les ressources sur lesquelles repose le tourisme.

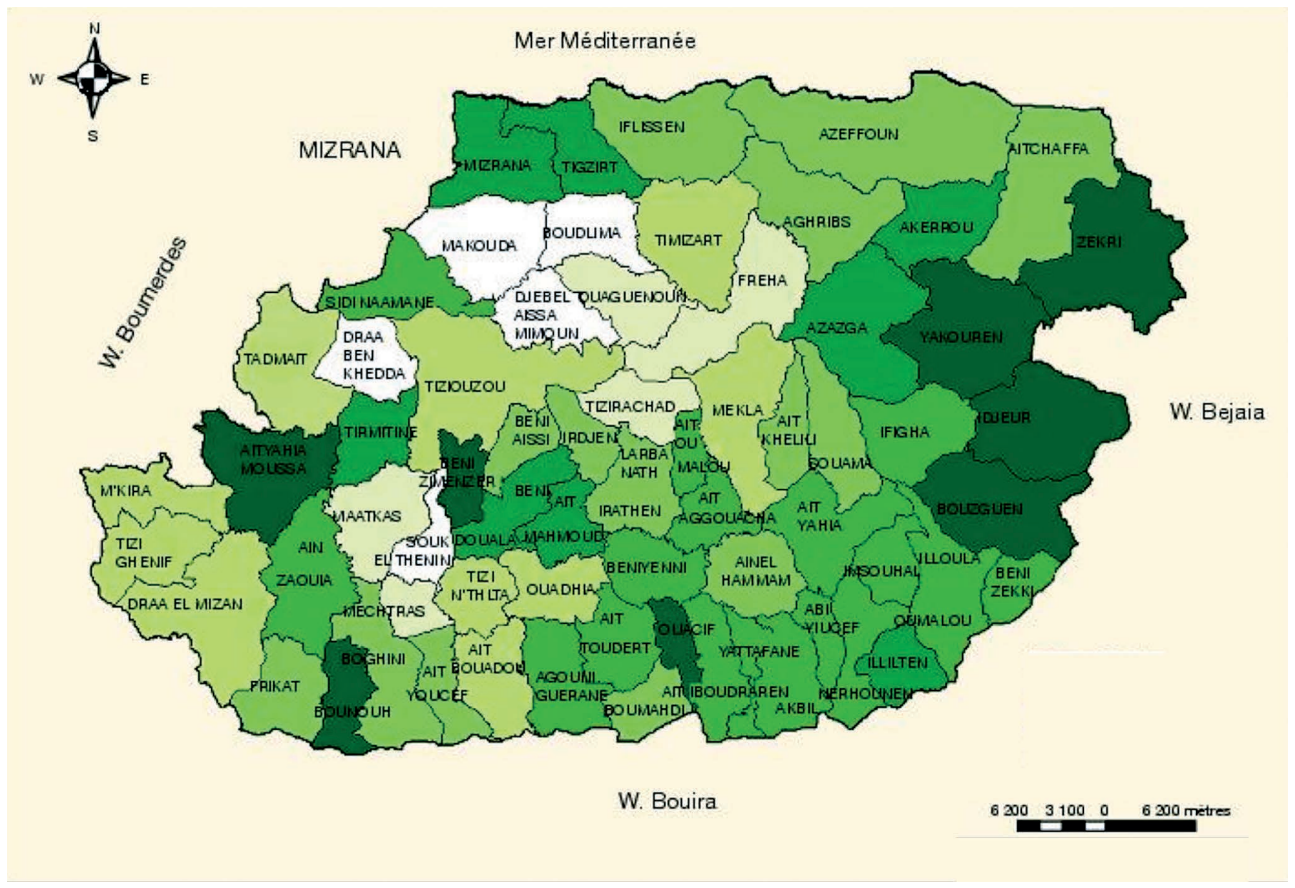

Fig. 1: Situation et découpage administratif de la wilaya de Tizi-Ouzou

Un tourisme durable repose sur trois composantes essentielles, la conservation des ressources potentielles quelles soient naturelles ou 
architecturales dont dépend la pratique du tourisme, l'amélioration des conditions de vie des populations locales et la satisfaction accrue du touriste. Fondé sur les principes de durabilité et d'équité, le tourisme permet l'amélioration des conditions de vie des populations montagnardes et l'accroissement de leurs enjeux.

\section{Espace montagnard de Kabylie du Djurdjura «Cas de Tizi-Ouzou»}

La wilaya de Tizi-Ouzou est limitée au Sud par la Wilaya de Bouira, à l'Est par Bejaia, à l'Ouest par Boumerdès et ouverte sur la méditerranée au Nord avec $85 \mathrm{~km}$ de côtes. Tizi-Ouzou occupe un territoire dont le relief est majoritairement montagneux fortement accidenté et s'étale sur une superficie de $2994 \mathrm{~km}^{2}$ qui se ventile sur 21 Daïras et 67 communes, elle fait partie de la Région Nord-Centre avec une charge démographique totale de 1127607 habitants. Selon le R.G.P.H 2008, Tizi-Ouzou en dépit de son urbanisation reste à dominance rurale avec un nombre de 349 agglomérations semi-rurales et rurales agglomérées se ventilant sur 45 communes et concentrant environ $59 \%$ de la population de la région.

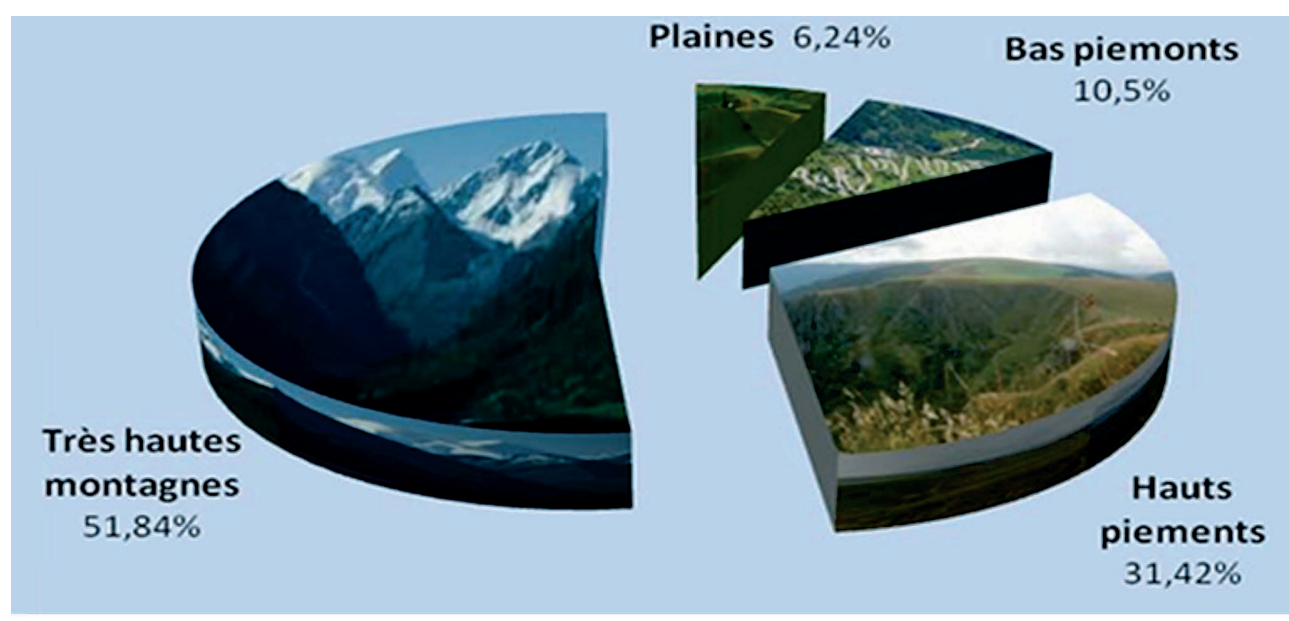

Source: APW de Tizi-Ouzou (ANDI 2013)

Fig. 2 : Régions physiques de la wilaya de Tizi-Ouzou

Bien que réduite en superficie, la Wilaya de Tizi-Ouzou présente un territoire morcelé et compartimenté du Nord au Sud où nous distinguons (05) cinq régions physiques dont : 
* La chaîne côtière qui se prolonge vers le massif Yakouren sans arrêtes vives et lignes de crêtes saillantes, soit un nombre total de 21 communes. * La vallée du Sébaou comprise entre la chaîne côtière et le massif central, paraît comme un estomac à l'envers. Elle a fait objet de convoitises depuis longtemps d'abord entre tribus ensuite avec les colons.

* Le massif central décrit par [Alain Mahé, 2006] comme étant la zone la plus prospère de toutes les Kabylies et abritant des densités fortes de populations qui font d'elle l'une des zones de montagnes la plus peuplée au monde, comprise entre 800 et 1200 mètres avec une dizaine de pics à 1500 mètres. La végétation est dense dont le couvert est plus arbustif. Ce massif est plus boisé que le Djurdjura mais beaucoup moins que la moitié orientale de la chaîne côtière.

* Les dépressions de Drâa-El-Mizan/Ouadhias comprises entre le massif central et les plissements du Djurdjura, c'est une plaine qui regorge 24000 hectares de terres cultivables.

* Le Djurdjura, souvent synonyme de Kabylie, dessine un arc dont l'orientation Est-Ouest et joue un rôle de barrage empêchant le contact des airs Sahariens du Sud et Océaniques du Nord.

\section{Des ressources patrimoniales à mettre en tourisme en Kabylie du Djurdjura}

La Kabylie du Djurdjura est peu propice à l'investissement et ce n'est qu'à travers le tourisme qu'elle peut aspirer au développement, elle constitue un environnement favorable abritant des richesses naturelles et patrimoniales, culturelles pouvant être un moteur pour la relance et le développement touristique de la zone de montagne. La promotion des activités touristiques permettra la valorisation et la sauvegarde du patrimoine tout en améliorant les conditions de vie des populations locales.

La région de la Kabylie, propose à travers ses ressources naturelles et ses villages perchés en montagne un énorme potentiel touristique à valoriser. Elle recèle un fort potentiel naturel et culturel faisant d'elle une destination privilégiée pour le tourisme de montagne qui peut être d'un apport considérable sur un développement touristique durable s'il est pris en charge comme il se doit. Sans oublier les richesses artisanales très variées dont bijouterie, robe kabyle, bois, tapis, poterie, ...etc. 


\section{1. La montagne du Djurdjura et ses curiosités}

Reconnue par l'UNESCO en 1997 comme une réserve de la biosphère, elle constitue un écosystème botanique, faunistique et climatique extrêmement précieux qui doit s'ouvrir aux scientifiques et au tourisme de la nature. Le parc national du Djurdjura crée en 1983 peut constituer une ressource importante de devise étrangère grâce à une clientèle européenne qui s'intéressera à la découverte d'une faune surprenante à une distance proche. Ainsi ce parc pourra considérablement participer à la promotion des activités rurales des villages implantés à sa périphérie. Le massif du Djurdjura compte plusieurs espèces de plantes d'animaux en voie de disparition, son relief est l'un des plus abrupts de l'Algérie, son plus haut sommet culmine les 2308 m d'altitude celui de «Lala Khadîdja ». La formation de ce massif remonte à environ 200 millions d'années, le Djurdjura renferme plusieurs curiosités naturelles esthétiques, d'une architecture orographique faites de pitons, de crevasses, de gouffres et de brèches. Le massif du Djurdjura synonyme de la Kabylie constitue un immense gisement qui abrite des pôles touristiques d'une infinie variété de produits.
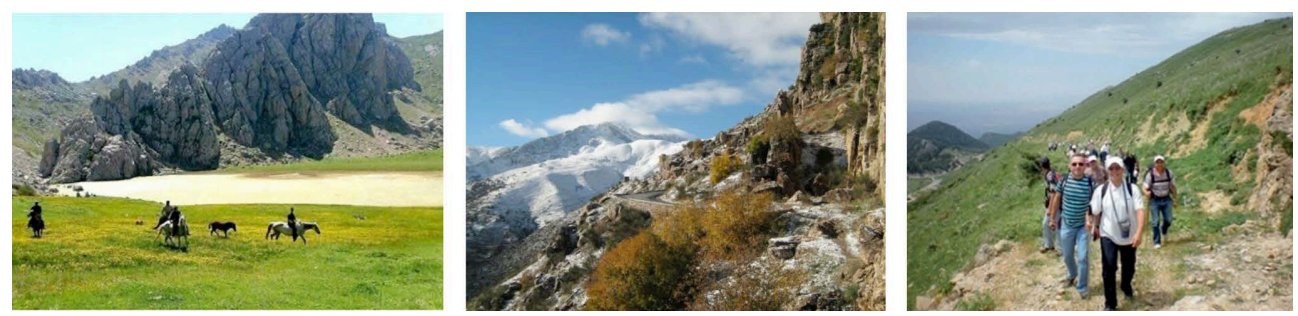

Photos 1, 2 et 3 - Sites naturels du Parc National du Djurdjura Source : http://www la-montagnedudjurdjura.com

Tala Guilef, Lalla Khedidja, le Lac d'Agoulmine, le Gouffre de Boussouil à 1259 mètres, les grottes du Macchabée, le Pic d'Azrou n'Thour, les cols de Tirourda à 1700 mètres et de Tizi-n'Kouilal à 1600 mètres sont autant de sites connus du Parc National du Djurdjura, favorables à la pratique du tourisme de montagne comme les sports d'hiver, les randonnées pédestres, l'alpinisme et la spéléologie, en plus des forêts très prisées de Yakouren et de Mizrana propices également pour les randonnées sportives, les séjours écologiques et culturels pour ses richesses faunistique et floristique. D'après la direction du Parc National du 
Djurdjura, ces sites accueillent chaque année plus de 500000 visiteurs, dont Tikjda avec 39\%, Tala Rana avec $29 \%$ et Tala Guilef avec $25 \%$, et cela est du à leur accessibilité par voies routières ainsi que l'existence d'infrastructures d'accueil assurant l'hébergement et la restauration.

\section{2. Les villages Kabyles traditionnels, un paysage culturel à valoriser}

En plus de ces fééries naturelles, les villages kabyles situés sur les deux versants du Djurdjura, viennent orner cette chaîne montagneuse avec leurs maisons entassées d'une beauté saisissante, l'architecture traditionnelle locale est sans doute un réceptacle crucial d'une activité touristique prometteuse, dont la prise de conscience de son importance a abouti à une reconnaissance officielle, tel le cas du village d'Ath el Kaid situé au versant Nord, perché sur un piton rocheux au cœur du Djurdjura. Un ancien village d'une valeur historique indéniable, témoin de différentes périodes de l'histoire du pays et de la région. Sa construction remonte à la période turque, et a continué à exister pendant des centaines d'années. Il a résisté aux affres de la colonisation française et semble défier les vicissitudes du temps et de la vie "moderne ». Il a grandement préservé son authenticité, remarquable par une beauté saisissante et un paysage fascinant. En application des dispositions de l'article 42 de la loi $\mathrm{n}^{\circ}$ 98-04 du 15 juin 1998 relative la protection du patrimoine culturel, ce village fut classé en 2009 et érigé en secteur sauvegardé dont le Plan Permanent de Sauvegarde et de Mise en Valeur du Secteur Sauvegardé est en cours d'étude.

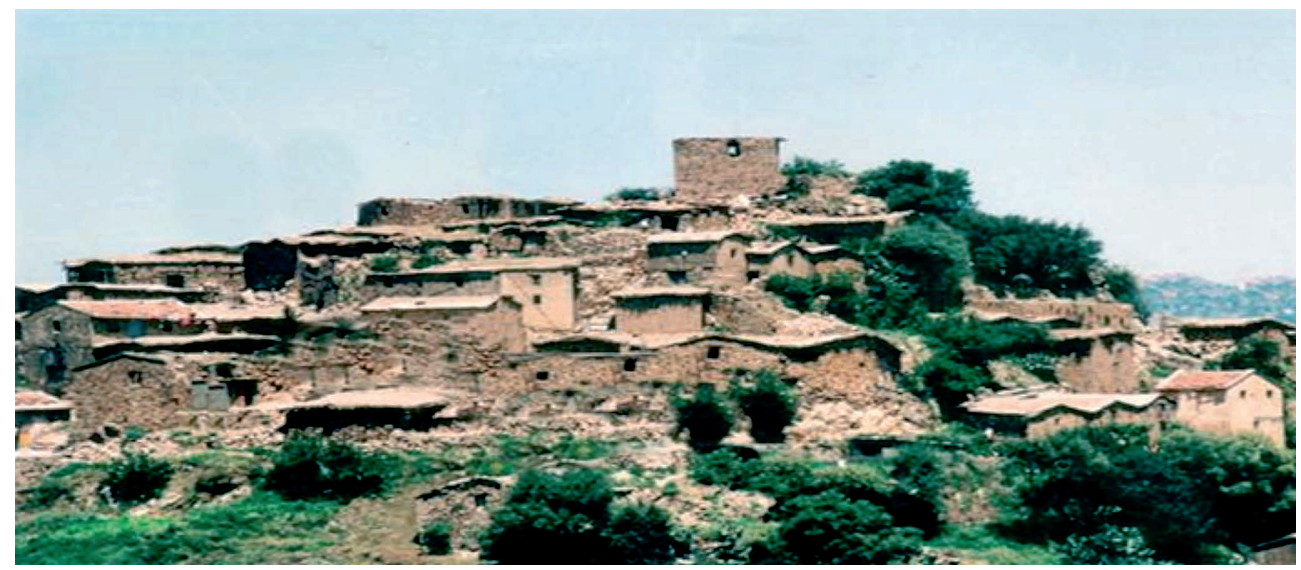

Photo 4 - Village d'Ait El Kaid / Source: PPSMVSS - Rapport de présentation 
Le village est essentiellement constitué de maisons traditionnelles anciennes, qui sont dans leur majorité abandonnées, sauf quelques familles qui y vivent encore.
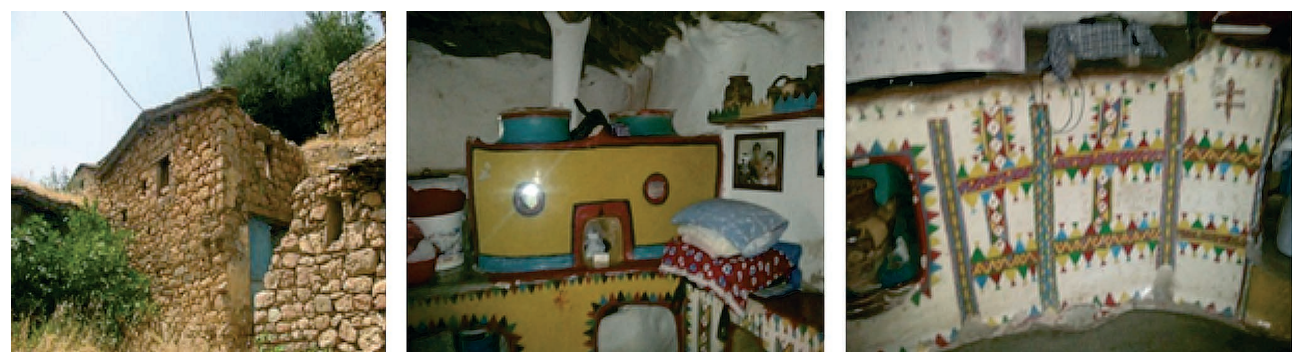

Photos 5: Vues intérieures d'une maison du village d'Ath El Kaid Source: Cliché Auteurs, juillet 2016

Le village en Kabylie n'est qu'une expression vivante des valeurs culturelles de ses habitants. C'est un espace chargé de significations qui peuvent se lire à toutes les échelles de l'espace villageois qui s'organise suivant des niveaux d'appartenance basés sur le principe de parenté et d'alliance. Généralement implantés sur des crêtes pour des préoccupations défensives, les villages kabyles sont composés d'un ensemble de ruelles et de maisons, d'une fontaine, d'une mosquée et de Tajmaat (espace d'assemblées).
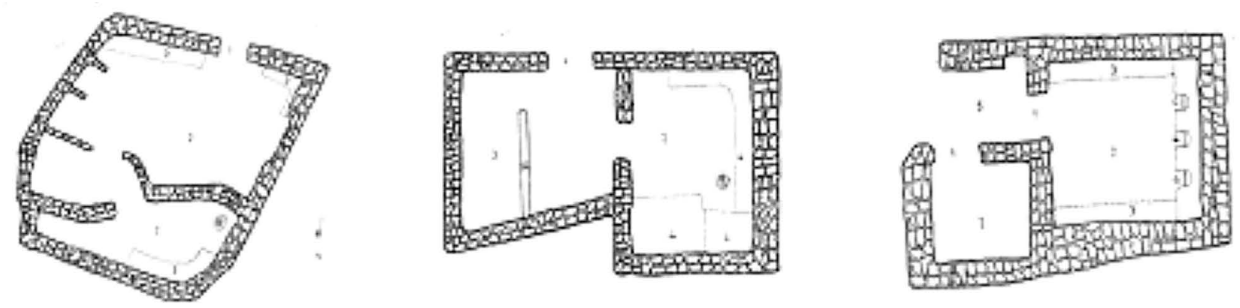

Fig. 3 : Plans de maisons du village d'Ath El kaid. / Source : DCW de Tizi-Ouzou (2007)

La maison d'Ath El Kaid préserve la distribution tripartite de la maison kabyle, comportant, Taquâat qui abrite le métier à tisser, Taaricht pour les provisions et Adaynin pour le bétail, ainsi que tous les éléments essentiels qui la composent. Elle est construite par les hommes. Sa poutre principale «Assalas almas » symbolise le chef de famille qui est chargé 
de sa protection, tan disque le pilier central« Tiguejdit » est le symbole de la femme qui est gardienne de la maison, préservatrice de l'honneur et des traditions.

La sensibilité des visiteurs n'est pas uniquement attirée par la splendeur de ses paysages naturels et des maisons traditionnelles, mais également par la diversité des activités humaines et leur originalité.

\section{3. L'artisanat, coutumes et savoir-faire à perpétuer}

La grande Kabylie, est une région très remarquable par ses produits artisanaux de qualité et d'une grande diversité. $\mathrm{Vu}$ les ressources modestes de cette terre de montagne, ses habitants ont perpétué un artisanat ancestral, expression d'un peuple artiste, qui vient soutenir leurs revenus ${ }^{1}$.

La région est aussi réputée pour la diversité, l'originalité et la qualité de son artisanat traditionnel qui couvre plusieurs métiers dont la bijouterie dans la région des Ath Yenni, la poterie dans les régions d'Ait-Kheir, Maatkas, Bounouh et Ouadhias, la tapisserie à Ait Hichem et l'ébénisterie, le tissage, la vannerie, la broderie dans plusieurs localités de la wilaya.

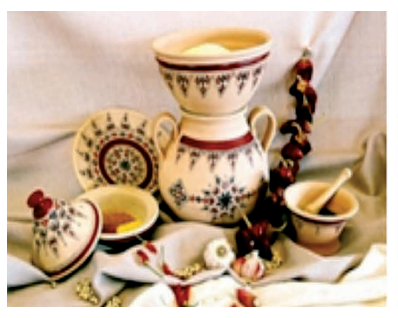

Photo 6 - Métiers artisanaux de la Kabylie du Djurdjura. Source :

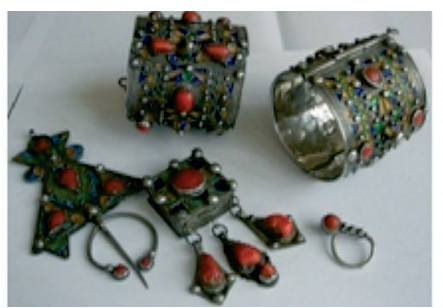

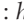

http://www-artisanatkabylie.com

Les techniques artisanales se transmettent de génération en génération. On peut distinguer deux des techniques artisanales exclusivement féminines qui produisent des objets destinés à des usages domestiques et celle d'artisans masculins spécialisés dont les produits sont d'une finition méthodique, destinés à la commercialisation. Les produits traditionnels sont une expression esthétique qui relève de l'art populaire

1 Le Tourisme Gisement Economique à Exploiter et à Valoriser, Direction de l'Artisanat de la wilaya de Tizi-Ouzou 
et de l'artisanat qui constitue un travail manuel spécialisé et rémunéré.

La Poterie : c'est l'activité la plus authentique de la Kabylie. La matière première utilisée est l'argile, les engobes blanches et rouge foncé, l'oxyde de manganèse noir-violacé. Les objets fabriqués sont destinés soit à l'usage domestique, soit à la commercialisation ${ }^{2}$.

Le Tissage : Le tissage est une activité féminine très répondue dans la région. C'est une activité familiale qui répond aux besoins domestiques. On produisait des Tapis, vêtements et couvertures, notamment le tapis d'Ath -hichem qui se distingue par sa qualité et beauté.

La Bijouterie : pratiquée par les hommes. La production est très variée, les bijoux fabriqués sont particuliers de par leurs couleurs. Les matières utilisées sont l'argent, le corail rouge, les clous de girofle marron « qrenfel », l'email «Nnil ». Des bijoux variés sont produits tel que : les colliers, les anneaux de chevilles "Akhelkhal », le bracelet "Ameclukh », les boucles d'oreilles, les fibules rondes ou triangulaires « Abzim ».

La Vannerie : une tradition industrielle de la région qui mérite d'être valorisée, dont la matière première est constituée essentiellement du roseau, et des fibres d'olivier sauvage. La vannerie en Halfa et rafia est pratiquée par les femmes, tandis que la vannerie en roseau est pratiquée par une main d'œuvre masculine appréciable. Plusieurs objets sont produits tel que : "Aqecwal », "Acwari », "Afarrug », "Dduh», etc.

Le costume traditionnel : L'art vestimentaire est d'un rôle déterminent dans la définition de l'identité kabyle, Le costume kabyle est porteur de signification et de symbole. L'habit féminin est composé de la robe traditionnelle, "L'fudha » ou "Thimehramth », "Amendil », et "Agous », elle se porte avec des bijoux traditionnels en argent. Les femmes continuent à porter la robe traditionnelle jusqu'à nos jours. Le costume masculin est composé du Bernus, "Taqecabit », "Aqendu »r, "Taamamt», "tacachit», "aserwual », et "Irkasen ». Les hommes ne portent plus le costume traditionnel, à part le "bernus » qui est un symbole d'honneur et de virilité. Il est porté dans les occasions et les cérémonies.

${ }_{2}$ Direction de l'Artisanat de la wilaya de Tizi-Ouzou, op. cit.1 
L'art décoratif : Les produits artisanaux, tel que poteries, tapis vêtements, sont ornés de motifs décoratifs très anciens. On retrouve également ces motifs surs les murs intérieurs de maisons kabyle. Ces décorations murales d'un caractère mystique sont l'œuvre de la femme, ils lui permettent de protéger son foyer des malédictions. Ils sont généralement réalisés avec un «Ousgou » mélange d'une roche de couleur ocre appelée «Amarghi » et une roche de couleur blanche "El madloug ${ }^{3}$

Les arts populaires : Les arts populaires en Kabylie sont riches et diversifiés, nous citons la dance, et les chants kabyles pratiqués dans des fêtes et occasions rituels. Les kabyles ont toujours accompagné leurs travaux de chants (ramassage du bois, cueillette des olives).

L'art culinaire : Le couscous est le plat caractérisant de la cuisine kabyle. Les femmes produisent la semoule roulée. L'art de rouler le couscous est un héritage artisanal légué précieusement et jalousement de mère en fille depuis la nuit des temps. La confection du couscous exige de la femme kabyle une grande patience. Toutes les graines doivent avoir le même calibre ${ }^{4}$.

Les fêtes populaires : Les fêtes populaires sont nombreuses et diverses en kabyles, elles sont soit saisonnières ou bien liées à des faits religieux. Nous citons parmi d'autres :

Yannayer : Qui est la fête du nouvel an berbère, pour la célébrer on organise «Imsil n yennayer » du couscous avec un coq sacrifié pour l'occasion. Tislit bu anzar: Destinée à faire tomber de la pluie en temps de sécheresse. Timecrret (Sacrifices partagés) : Associés le plus souvent à des occasions telles que «l'Aid el Adhha », ils sont organisés par tadjmaat. Tizwizi : L'entraide est une tradition dans la communauté villageoise kabyle. Elle s'imposait à chaque fois que quelqu'un s'engage dans un travail difficile, tel que la construction $\mathrm{d}^{\prime}$ une maison ${ }^{5}$.

\section{4. Une agriculture de terroir : un emblème d'une vie paysanne}

La productivité dans la Kabylie du Djurdjura est constituée

\footnotetext{
3 Témoignage de Na tassadit op. cit.

4 Le Tourisme Gisement Economique à Exploiter et à Valoriser, direction de l'artisanat de la wilaya de Tizi-Ouzou

5 Idem
} 
essentiellement des produits du terroir entre autres, les figues, les olives et l'huile d'olives.
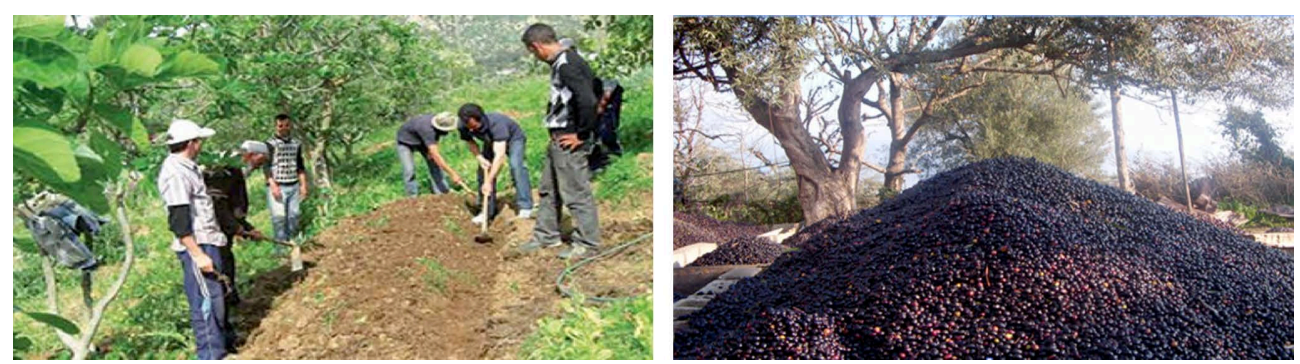

Photo 7-8: Arboriculture et la twiza(entraide)

Source : agriculture de montage en Algérie collection dossier agronomique

L'olivier: cet arbre emblématiques de la région tant au niveau économique que culturel, est surtout cultivé pour la production d'huile d'olive (zzit uzemmur). Celle de Kabylie est réputée pour être une des meilleures du bassin méditerranéen, elle est la seule au monde qui satisfait les critères d'une huile d'olives $100 \%$ biologique. Elle présente plusieurs variétés, elle est utilisée dans différents domaines tel que la médecine traditionnelle, la médecine moderne ainsi que l'industrie cosmétique.

Le figuier: Le figuier constitue également une richesse et un symbole de la région, Il fait partie de son histoire, une culture qui revêt une importance sociale et économique fondamentale. Extrêmement productif, cet arbre majestueux et son fruit symbolisent souvent la richesse et la fécondité. Le patrimoine figuicole recèle une grande diversité de produit de qualité, et $\mathrm{l}^{\prime}$ Algérie occupe la cinquième place concernant la production avec 70000 tonnes de figues, la majorité de la production est fournie par les régions de montagne de Kabylie.

\section{Le tourisme patrimonial-culturel véritable moteur de développement}

Dotées de particularités naturelles et de patrimoine culturel qui en font des endroits touristiques indéniables, les montagnes sont à la fois des lieux de diversité biologique et culturelle. C'est cette spécificité qui a pu renforcer leur position « entre paysage naturel et paysage culturel ». En dépit de tous ces lieux de beauté splendide à la valeur touristique établie, aucun intérêt ne leur est manifesté à présent par les pouvoirs 
publics. Contrairement au Maroc qui a une avancée en la matière surtout en rapport au projet concernant les espaces ruraux dont une stratégie globale de mise en tourisme participatif a été enclenchée touchant plusieurs villages à travers le pays. Les dépenses des visiteurs ont des impacts directs et indirects sur les économies locales en termes de revenus, de recettes en devise, d'emploi, d'épanouissement de l'artisanat, de valeur ajoutée, d'investissement, de production et de perspectives commerciales. Le tourisme et la culture contribuent tout autant à la diversification des activités économiques qu'au développement social, culturel et à l'attractivité de cet espace, ils sont de ce fait des moteurs essentiels du développement dont les défis et perspectives se résument dans ce qui suit :

- Valorisation de la beauté unique du paysage pour un tourisme doux

- Intégration de l'artisanat et de la gastronomie pour le développement de l'identité culturelle et d'un tourisme proche de la nature (marchés paysans, label pour les produits de l'espace et $\mathrm{du}$ terroir tels miels, figues, olives etc...). La promotion de l'agriculture notamment la filière de l'oléiculture et celle de la figuicole doit s'appuyer sur des potentialités spécifiques de la région dont le savoir-faire local afin de ne pas réduire le souci de sa valorisation seulement à sa rentabilité économique mais également à sa dimension patrimoniale.

- Promotion de l'offre culturelle par des thématiques porteuses et attractives

- Promotion des échanges entre tous les acteurs du tourisme et de la culture

- Création de Parcs naturels et paysagers

- Installation d'entreprises touristiques très compétitives

\section{Conclusion}

Nous ne pouvons parler de sauvegarde durable d'un patrimoine que s'il est intégré à la vie de tous les jours des populations, en y vivant, en l'entretenant et en le valorisant par sa mise en tourisme. En effet, le patrimoine dans toutes ses facettes peut être un instrument de développement économique et de promotion territoriale de la montagne essentiellement dont la valorisation touristique est source de revenu multiple et créatrice de postes d'emploi. Le territoire comme le 
patrimoine confèrent aux réalités sociales une consistance matérielle, à travers les signes et les symboles investis dans des objets, des lieux et des paysages. Il semble intéressant de s'inspirer des principes et approches du tourisme durable qui se fonde sur la promotion d'activités économiques viables qui respectent l'environnement et qui permettent l'intégration sociale des populations locales. La dimension économique est essentielle en zone de montagne qui doit impérativement valoriser ses propres ressources et trouver des revenus complémentaires. Au-delà des activités artisanales, c'est bien le tourisme qui aujourd'hui apparaît comme une nouvelle source de revenus potentielle pour les habitants. La nécessité de forger une identité et une image communes à travers des actions de vulgarisation et de diffusion pour toute la zone de montagne Kabyle est plus qu'indispensable, au vu des opportunités qu'elle présente telles :

- Attractivité pour les séjours de détente, pour le tourisme de montagne et de bien être

- Fort potentiel culturel, historique et patrimonial par les ressources précieuses en espace naturel, richesse et diversité du paysage et de l'architecture

- Notoriété importante de l'espace

- Potentiel de développement du tourisme rural, vert et sport (balade, parapente, alpinisme...)

En conclusion, le patrimoine culturel matériel et immatériel pourrait constituer une base solide pour des actions de développement économique et socioculturel. Le développement devrait être pensé dans un rapport étroit avec la continuité et la permanence de certains caractères identitaires, afin de les préserver et de concevoir leur évolution dans le cadre de leurs spécificités identitaires.

\section{Bibliographie}

- Ahmed Saber. La protection des zones de montagne dans le cadre du développement durable, Aménagement du territoire, Le Maghreb 31/08/2008

- Ait-lhadj Zoulikha. Développement territorial, patrimoine et identité : quels défis pour le paysage d'Ain El Hammam ?, Colloque Ressources Territoriales et Développement : avancées théoriques et expériences

- Berque A. 2000. Introduction à l'étude des milieux humains, Ecoumène, Paris Berlin 
- "Territoire en France les enjeux économiques de la décentralisation ", Economica, Paris, 1984, P146

- de terrain, Tizi-Ouzou, les 17, 18 et 19 novembre 2014

- Akkache-Maacha D. Art et artisanat traditionnels de Kabylie, Revue Campus Université Mouloud Mammeri Tizi-Ouzou

- Avocat C. Actes du Colloque : Lire le paysage, lire les paysages, 24-25 Novembre 1983, Université de Saint-Etienne

- Bart F., Morin S. \&Salomon J. N. 2001. Montagnes tropicales : identités, mutations, développement, DYMSET, $\mathrm{n}^{\circ} 16,671 \mathrm{p}$.

- Bourdin A. 1996. Sur quoi fonder les politiques du patrimoine urbain; professionnels et citoyens face aux témoins du passé, les annales de la recherche urbaine $n^{\circ} 72$, p.p.6-7

- Bras C., Le Berre M., Sgard A. 1984. La montagne, les géographes et la géographie, RGA, n²-3.

- Chiva I. 1994. Une politique pour le patrimoine culturel rural, Rapport présenté à Mr. Jacques Toubon, Ministre de la Culture et de la Francophonie

- Comité du Tourisme l'OCDE (2009). The Impact of Culture on Tourism, French translation, Centre Entrepreneurship SMEs and Local Développement

- Debarbieux B.1995. Tourisme et montagne, Economica, Paris

- Di Meo G. [S.D]. Processus de patrimonialisation $\mathcal{E}$ de construction des territoires http://www.ades.cnrs.fr/IMG/pdf/GDM_PP_et_CT_Poitiers.pdf

- Di Meo G. 2007. Identités et territoires: des rapports accentués en milieu urbain ?, METROPOLES, n¹, p.p.69-94

- Dower M. La Ressource Patrimoine : Un atout pour le développement local, [http:/ / ec.europa.eu/agriculture/rur/leader2/rural-fr/biblio/herit/art01.htm]

- Greffe X.1984. Economie des politiques publiques, Dalloz, Paris

- Idir M. S. Valorisation du patrimoine, tourisme et développement territorial en Algérie : cas des régions de Bejaia en Kabylie et Djanet dans le Tassili n'Ajjer.

- Khelil A. 2000. La société montagnarde en question, ANEP

- Mahé A. 2006. Histoire de la Grande Kabylie XIX-XX Siècles, Bouchène

- Mekati H. 2013. Dans quelle mesure le tourisme en zone de montagne peut-il constituer au développement local de la Kabylie ?, mémoire Master, faculté des sciences économiques, commerciales et sciences de gestion, UMMTO.

- Morizot J. 2001. Les Kabyles : propos d'un témoin, 1'Harmattan

- Nait Djoudi O.1994. Kabylie : un espace agraire en recomposition, CNRS, Université Paul Valéry, Montpellier III

- Ollagnon H. 1984. Acteurs et patrimoine dans la gestion de la qualité des milieux naturels, Aménagement et Nature, $\mathrm{n}^{\circ} 74$.

- Poullaouec-Gonidec P. Vivre E habiter le paysage, Actes du Colloque du 7 - 8 
Juin 2001, Rabat-Maroc

- Prenant A. 1980. La mutation en cours des modes de croissance urbaine en Algérie, Hérodote $\mathrm{N}^{\circ} 17$, 1er trimestre.

- Sacareau I. 2003. La montagne, objet géographique, Belin Supérieur

- UNESCO, Tourisme, culture et développement dans la région arabe

- Yesguer H. 2008. Les incidences du système de transport sur l'ouverture des zones rurales isolées. Le cas de la Kabylie (Algérie), les Cahiers Scientifiques du Transport $\mathrm{n}^{\circ} 54$, p.p.125-146

-Salhi Med B. in Messaci-Belhocine N. (dir.). Espace montagnard entre mutations et permanences, CRASC, p.p.11-50 